\title{
Editorial: Introducing Physical Review Research
}

It is with great pleasure that I present the first issue of Physical Review Research, an international, open access journal published by the American Physical Society (APS) in direct support of its mission to advance and diffuse the knowledge of physics for the benefit of humanity, promote physics, and serve the broader physics community.

We are launching Physical Review Research to achieve three main objectives: (1) to cover the entire range of topics in physics and related fields, including interdisciplinary and newly emerging areas of research, (2) to offer an attractive option for all authors who prefer or are required to publish in fully open access journals, and (3) to align its quality standards and selection criteria with those of the other long-established topical journals in the family, such as Physical Review A-E, Physical Review Applied, Physical Review Fluids, and Physical Review Materials.

To effectively cover the topical breadth and geographic range of this multidisciplinary, international peer-reviewed journal, we have committed to appointing a diverse and global team of esteemed active researchers to serve as Lead Editors and Editorial Board members. I am extremely pleased to announce that Professors Raissa D'Souza, Jian-Wei Pan, and Nicola Spaldin have agreed to serve as the founding co-Lead Editors of Physical Review Research and represent the journal within their respective regions and research communities.

Raissa D'Souza is a Professor at the University of California, Davis, Departments of Computer Science and Mechanical and Aerospace Engineering. She is widely recognized for her interdisciplinary research in network science and complex systems. D'Souza is a Fellow of APS, a National Academy of Sciences Kavli Fellow, and Outgoing President of the Network Science Society (NetSci). Her honors include the inaugural NetSci Euler Prize and the Test of Time Award from the Association for Computing Machinery.

Jian-Wei Pan is a Professor of Physics at the University of Science and Technology of China (USTC) in Hefei, China. He is an international leader in the rapidly growing interdisciplinary field of quantum science and technology. Pan is an Academician of the Chinese Academy of Sciences (CAS) and an APS Fellow. His honors include the First Prize of National Prize for Natural Sciences of China, the Future Prize in Physical Science, and the OSA R. W. Wood Prize.

Nicola Spaldin is a Professor of Materials Theory at the ETH Zürich, Switzerland. In her research she develops and applies electronic structure methods to understand and design materials with coupled and competing electronic, magnetic and structural instabilities. Her honors include the APS McGroddy Prize for New Materials and the L'Oréal-UNESCO For Women in Science award. She is a Fellow of the APS, a Fellow of the Royal Society, and Foreign Member of the National Academy of Engineering.

Despite residing in distinct regions and research spaces, all three share the vision encompassed by our first objective: to cover high-quality research from all areas of interest to physicists, and thereby provide researchers the opportunity to share their results among a broad audience.

The second and third main objectives of the journal to offer a fully open access journal while maintaining editorial rigor and high quality is nicely summarized by Nicola in her first email to me in our correspondence about the launch of Physical Review Research: "As more funding agencies require publication in 
open access-only journals it's great to have this option within the Physical Review. Many researchers were getting worried that we would only have a very limited set of journal options."

While Physical Review Research has distinct Lead Editors and Editorial Board, to ensure we deliver the experience and quality authors value, trust, and expect from any Physical Review title, submissions to this new journal are being handled through a collaborative effort across the same editorial team of Ph.D. scientists and professional support staff who manage the peer review for all of the Physical Review journals.

All of us at APS who are involved with the Physical Review journals care deeply about their ongoing significance to science, are excited about this new endeavor, and are dedicated to delivering the excellence our communities expect from us via this new member of the journal family. We hope that Physical Review Research will initiate conversations across traditional boundaries, reveal new opportunities for collaboration, and facilitate future discoveries, and thus directly support the aim of the APS to advance scientific discovery and research dissemination.

(D) Published 9 August 2019

Michael Thoennessen Editor in Chief

DOI: 10.1103/PhysRevResearch.1.010001 